\title{
Appareil
}

$20 \mid 2018$

Arts contemporains et patrimoine

\section{Les Songes de la nef, pour une " prise de site " musicale et sonore}

Alain Bonardi

\section{OpenEdition}

1 Journals

Édition électronique

URL : http://journals.openedition.org/appareil/2873

DOI : 10.4000/appareil.2873

ISSN : 2101-0714

Éditeur

MSH Paris Nord

Référence électronique

Alain Bonardi, «Les Songes de la nef, pour une « prise de site » musicale et sonore », Appareil [En ligne], 20 | 2018, mis en ligne le 29 octobre 2018, consulté le 30 juillet 2020. URL : http://

journals.openedition.org/appareil/2873; DOI : https://doi.org/10.4000/appareil.2873

Ce document a été généré automatiquement le 30 juillet 2020.

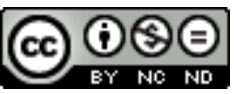

Appareil est mis à disposition selon les termes de la Licence Creative Commons Attribution - Pas d'Utilisation Commerciale - Pas de Modification 4.0 International. 


\title{
Les Songes de la nef, pour une " prise de site » musicale et sonore
}

\author{
Alain Bonardi
}

Je remercie Martine et Jean-Louis Déotte, Caroline Coppey, Frédéric Roussel, Jean-Pierre Peter et Quentin Nivromont.

\section{Introduction}

1 Ce texte revient sur la conception et la mise en œuvre d'une installation sonore pour l'Hôtel-Dieu de Tonnerre en Bourgogne intitulée Les Songes de la nef'. Double hommage au compositeur-chercheur Jean-Claude Risset (1938-2016) et au philosophe Jean-Louis Déotte (1946-2018), elle est fondée sur un dialogue avec leurs écrits. Cet article revient sur ces échanges, en particulier celui avec Jean-Louis Déotte, engagé de son vivant en 2017 et poursuivi après son décès autour de ses textes dans le cadre de notre proposition artistique ${ }^{2}$.

2 Le point central du dialogue avec Jean-Louis Déotte est la notion de "prise de site ", que l'on voit apparaître sous sa plume en dialogue avec l'architecte Jacques Boulet, pour remplacer celle de «in situ» finalement «trop vague, donnant lieu à trop d'expositions hétérogènes ${ }^{3}$ ». Le philosophe évoque d'abord la notion de prise de site dans le cadre de l'Hôtel-Dieu de Tonnerre : « Il s'agit de distinguer ce qui a eu lieu et aura lieu dans l'Hôtel-Dieu - depuis les premières installations de Caroline Coppey, Didier Guth, Germain Roesz (2014-2015-2016) - de ce qui pourrait avoir lieu dans un espace comme la Pharmacie où les œuvres n'enchaînent pas sur l'espace ${ }^{4}$. " Il apparaît que la notion est généralisable à toutes les situations proposant un accrochage d'œuvres contemporaines dans des lieux dits patrimoniaux; selon Jean-Louis Déotte, ces derniers ont été préalablement mis sous vitrine, sont devenus des monuments sans destination effective, pour en isoler la valeur esthétique. Pour reprendre une notion qui lui est chère, l'Hôtel-Dieu est devenu un « suspens » comme le sont des tableaux dans un musée : 
[...] la question de l'art n'est possible que du fait de l'institution de cet appareil spécial qu'on appelle musée, parce qu'il suspend, met entre parenthèses, la destination cultuelle des œuvres, c'est-à-dire leur capacité esthétique de fairecommunauté et de faire-monde et qu'à partir de lui les œuvres devenant des suspens peuvent être pour la première fois contemplées esthétiquement pour ellesmêmes, à condition, comme le signale Benjamin, que je reste à trois mètres d'elles ${ }^{5}$. proposées dans ces lieux patrimoniaux : seront-elles réduites à n'être que de simples "suspens" absorbés dans un "suspens " plus vaste ? C'est là que la "prise de site " prend tout son sens, proposant une relation dynamique entre les œuvres et le lieu. En posant que les œuvres contemporaines devraient faire la «critique ${ }^{6} » d u$ lieu patrimonial qui les reçoit, Jean-Louis Déotte refuse toute proposition de type muséal mais aussi toute proposition de type "décoratif»: le lieu patrimonial n'est pas une salle de musée et n'a pas plus besoin d'être orné par des œuvres contemporaines.

Pour un musicien, cette notion de "critique» évoque immédiatement celle de « commentaire » établie par le compositeur italien Luciano Berio (1925-2003) dans sa fameuse citation : «Le meilleur commentaire que l'on puisse faire d'une symphonie ou d'un opéra, c'est d'écrire une autre symphonie, un autre opéra ${ }^{7}$. " Toutefois la notion de «critique » telle que l'entend Jean-Louis Déotte généralise l'idée de commentaire en ouvrant à la constitution d'enchaînements entre les œuvres ou les arts visant l'enrichissement.

\section{Composer veut dire : construire un instrument}

Mais comment une œuvre musicale ou sonore peut-elle venir "critiquer» ou « commenter » un ouvrage patrimonial comme l'Hôtel-Dieu? Quelles « hypothèses » de ce bâtiment une composition peut-elle tenter d'interroger? Comment "amplifier " telle ou telle potentialité du lieu? Lors des premiers pas dans la conception de l'installation Les Songes de la nef, ces questions sont vite apparues dans le dialogue engagé avec Jean-Louis Déotte et Caroline Coppey au sein de l'Association APHT (Art et Patrimoine Hospitalier en Tonnerrois) et lors des premières visites de l'Hôtel-Dieu à Tonnerre.

6 Notre proposition d'installation est fondée sur la notion d'instrument, et s'inspire de la fameuse citation du compositeur allemand Helmut Lachenmann (né en 1935): " composer veut dire : construire un instrument ${ }^{8} »$. Dans cette conception, l'œuvre est d'abord un instrument à construire sur mesure avant de devenir une partition. L'idée ne provient pas uniquement de la lecture de Lachenmann; ses origines sont également à chercher dans les caractéristiques du lieu : par ses dimensions, par le dépouillement de son architecture, par la simplicité géométrique de ses lignes, par l'absence de piliers expliquant une acoustique particulière avec une résonance longue, claire et peu brouillée, le bâtiment suscite des questions similaires à celles que se posent les organistes, notamment : comment « jouer » l'Hôtel-Dieu pensé comme un instrument? Dans un premier temps, nous nous sommes projetés dans la situation d'un facteur d'orgue qui aurait à concevoir un instrument pour le lieu. Ce qui nous rapproche encore davantage de la pensée de Lachenmann, qui fait référence à l'orgue comme modèle même de la composition ${ }^{9}$ :

[...] comme il s'agit avec la construction d'un tel instrument d'une exploration de

différents niveaux et couches reliés entre eux [...], je m'en tiens finalement à 
l'image d'un orgue imaginaire, assemblant différents manuels, certains courts, d'autres plus étendus, les uns qui font beaucoup d'effet, les autres statiques ou mobiles, ressortant plus ou moins $[. . .]^{10}$.

7 L'image d'un orgue imaginaire nous semble très pertinente et a inspiré l'installation électroacoustique. Il s'agit de faire sonner l'instrument en faisant sonner le lieu. Que ce soit un orgue ou un ensemble de haut-parleurs, l'instrument est tout autant le dispositif producteur de sons que le lieu qui l'accueille. Ainsi, nous avons souhaité construire un instrument «Hôtel-Dieu » au-delà d'un dispositif de haut-parleurs. Ce dernier devient alors unique, fait sur mesure pour le lieu, tout le contraire d'un dispositif générique que l'on pourrait installer dans différents lieux patrimoniaux.

\section{L'acoustique comme cadre opératoire de la prise de site}

Dans cette approche, l'acoustique prend toute son importance et devient le cadre conceptuel et opératoire permettant de penser l'installation dans son ensemble comme dans les détails, depuis la création du contenu sonore jusqu'à la mise en espace dans l'Hôtel-Dieu et aux interactions entre les sons et le lieu. L'acoustique est donc envisagée ici selon plusieurs approches qui sont évidemment liées, de la genèse synthétique du son électronique à sa diffusion dans l'espace et enfin la mise en résonance de l'HôtelDieu devenu instrument.

\subsection{Modèle de synthèse du son électronique}

9 Du point de vue du calcul sur ordinateur des sons joués, le point de départ est un modèle de synthèse d'un son de cloche proposé par le compositeur Jean-Claude Risset ${ }^{11}$, dont les investigations dans le champ du sonore ont transformé la composition musicale grâce à des procédés informatiques apparus au $\mathrm{xx}^{\mathrm{e}}$ siècle. Dans son catalogue de sons synthétisés par ordinateur ${ }^{12}$ publié en 1969, Risset s'est intéressé à la compréhension, la modélisation et la synthèse de sons complexes: dans le cas de la cloche, des mesures et observations physiques ont permis à Risset de modéliser ce son complexe comme somme de 11 sons élémentaires, des sinusoïdes, dont les fréquences, du grave à l'aigu, ne sont pas échelonnées régulièrement: il s'agit d'un son inharmonique.

Risset donne également leur amplitude temporelle, c'est-à-dire l'attaque des composantes et leur extinction dans le temps; il retrouve dans sa modélisation ce que chacun peut percevoir en écoutant une cloche : l'aigu du son disparaît beaucoup plus vite que le grave. Le spectrogramme ci-dessous montre les 11 composantes calculées sur la base d'une fréquence de $440 \mathrm{~Hz}^{13}$. Les fréquences sont données selon l'axe vertical (de bas en haut, du grave à l'aigu), et le temps selon l'axe horizontal. 


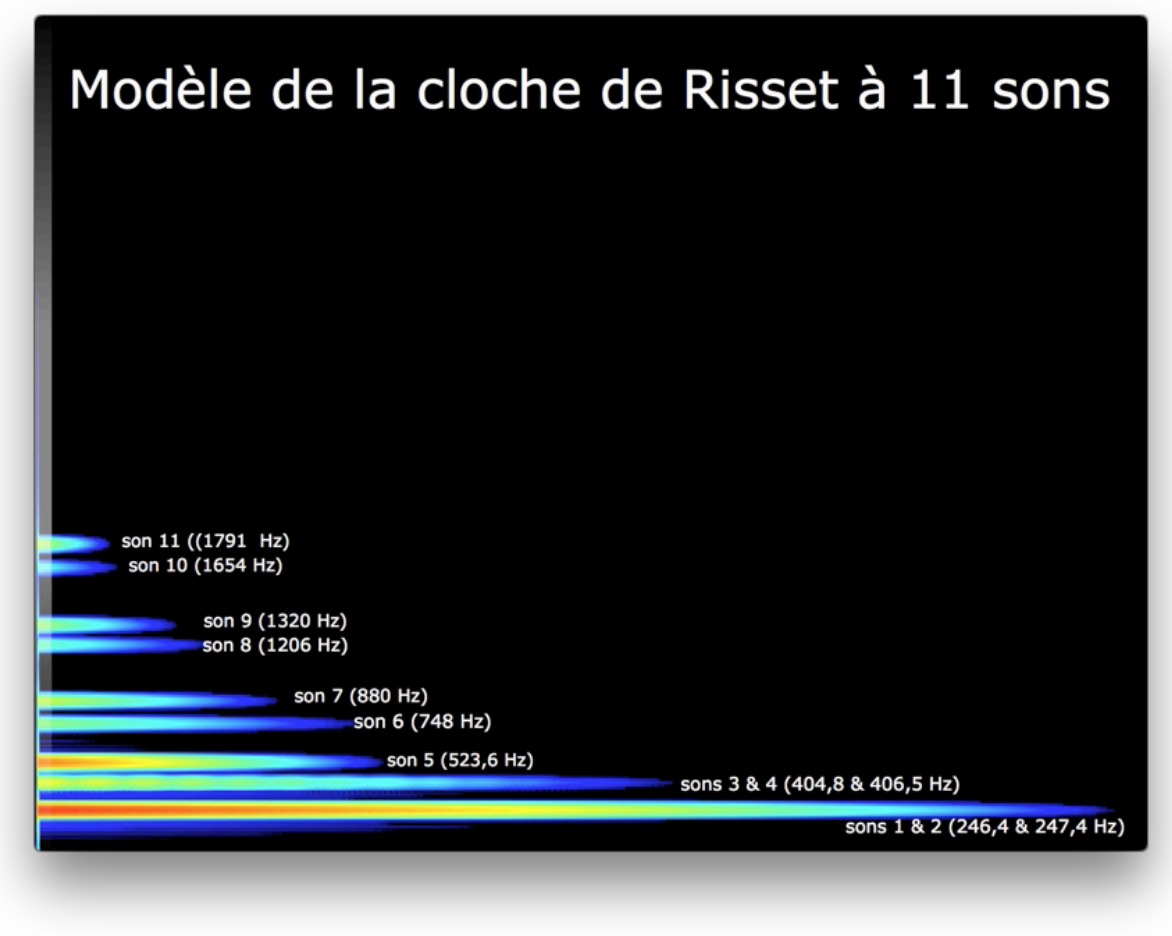

Source : auteur

11 Cette modélisation de cloche a été notre point de départ pour penser l'espace de la nef comme mis en résonance par une grande cloche composée de multiples cloches plus petites, entièrement calculées par ordinateur et diffusées sur un ensemble de hautparleurs. Nous avons étendu le modèle de Risset de manière récursive : le principe de superposition de 11 sons est conservé, mais ces 11 sons ne sont plus des sinusoïdes élémentaires : ce sont des sons de cloches, eux-mêmes composés de 11 sons. D'où le principe d'une cloche de cloche, comportant 11 fois 11, c'est-à-dire 121 sons qui seront envoyés vers les haut-parleurs. C'est avec cette palette de 121 fréquences élémentaires que nous avons imaginé 11 séquences sonores d'une durée totale de 88 minutes, qui sont jouées par un ordinateur.

12 Ces sons, joués ensemble ou décalés selon des intervalles temporels allant de quelques millisecondes à plusieurs secondes, permettent de créer des polyphonies. Lorsque les 121 sons sont regroupés, l'installation produit des timbres qui suggèrent différents instruments, comme des cloches ou des voix. Lorsqu'ils sont éparpillés, les sons sont diffusés comme dans un tintinnabulement. Le spectrogramme ci-dessous montre un exemple de "pulvérisation » des 121 sons dans la $1^{\text {re }}$ séquence. 
Figure 2. Spectrogramme du début de la première séquence

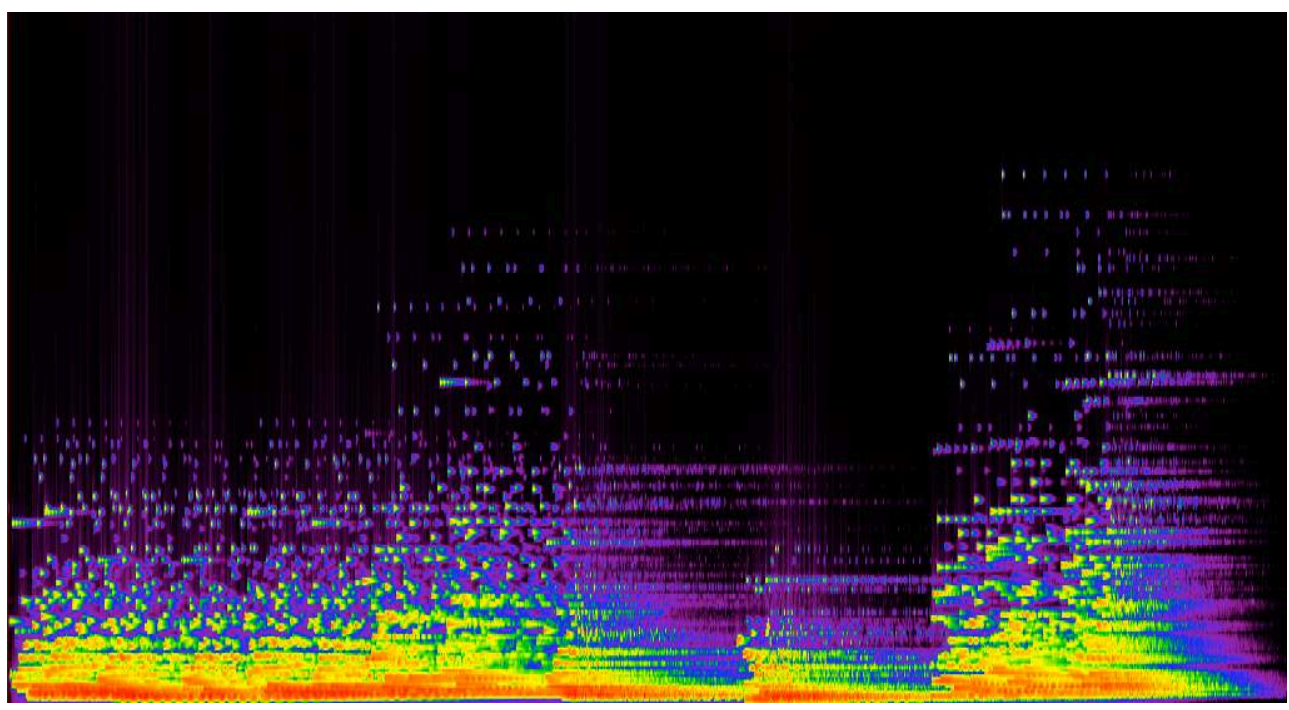

Source : auteur

\subsection{Diffusion spatiale de la cloche de cloche}

Dans la nef, cette cloche de cloche à 121 composantes sonores est diffusée par 9 hautparleurs étageant les sons du grave à l'aigu. Le schéma ci-dessous montre l'implémentation des 9 sources sonores indiquées par des chiffres entourés d'un cercle. Les haut-parleurs sont suspendus aux poutres en bois de l'Hôtel-Dieu. Les sons les plus graves sont émis par le haut-parleur 1, les sons les plus aigus par le haut-parleur 9. Nous avons choisi d'échelonner les sons du grave à l'aigu en allant du chœur à la tribune (de droite à gauche sur le plan ci-dessous). Nous avons symboliquement associé le chœur côté est aux fréquences graves, fondements des sons complexes, et la tribune côté ouest aux fréquences aiguës.

Figure 3. Implémentation des haut-parleurs dans l'Hôtel-Dieu

aigu

grave

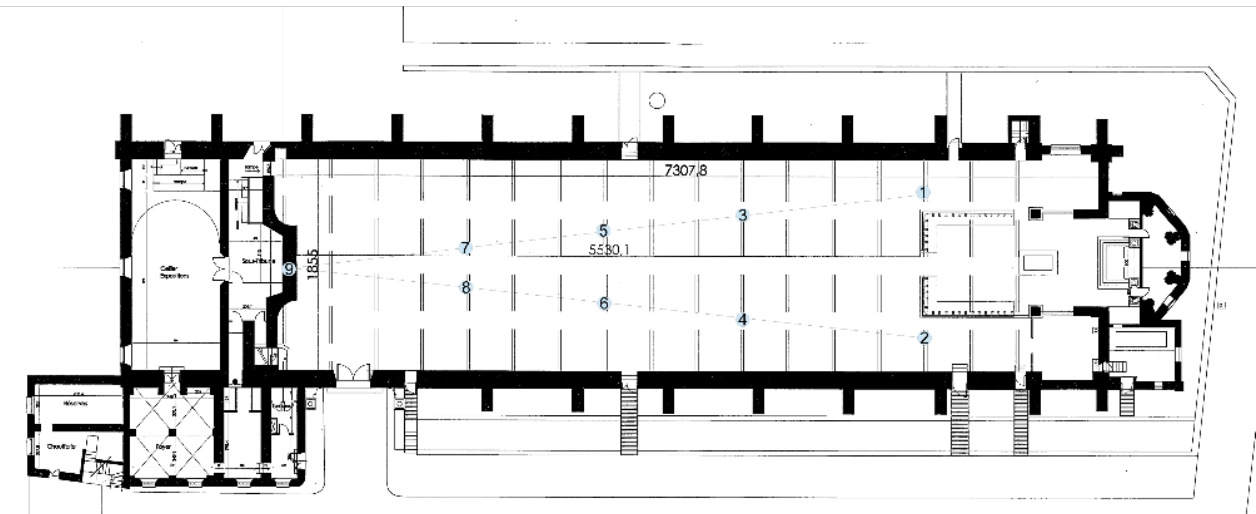

Source : auteur

Dans le cas de sons élémentaires comme des sinusoïdes, la physique permet aisément d'associer des longueurs à des fréquences, notamment des rapports de longueurs à des rapports de fréquences. Si l'on considère les longueurs d'onde et les fréquences, les 
rapports varient en sens inverse: lorsque l'on monte d'une octave, du la $a_{3} a_{1 a_{4}}$ par exemple, la fréquence est multipliée par 2, alors que la longueur d'onde est divisée par 2. Les tuyaux d'un orgue, pour revenir à cet instrument, montrent cette relation. Dans le cas de notre installation, cette mise en correspondance pourrait être intéressante pour calculer les distances entre haut-parleurs en connaissant les fréquences qu'ils émettent.

Cette approche n'est évidemment pas directement possible avec le système de synthèse retenu, sur la base de 121 sons sinusoïdaux, puisque 9 haut-parleurs seulement sont utilisés. Nous avons donc opté pour une approche statistique en reliant des paramètres macroscopiques de contrôle de la synthèse à des paramètres de positionnement des haut-parleurs comme le choix des poutres d'accrochage des haut-parleurs ou la distance séparant les sources 1 et 2 . La figure 4 montre les paramètres de contrôle de la synthèse utilisés tels qu'ils apparaissent dans le logiciel de synthèse des sons ${ }^{14}$. Par exemple, le paramètre "delay max " qui apparaît au milieu de l'écran indique le maximum du retard des 121 sons : chaque son sera retardé d'une durée tirée au sort comprise entre 0 et 5 secondes ( 5000 millisecondes). Il peut être intéressant de relier ce paramètre à celui de spatial dispatching qui contrôle globalement la répartition des 121 sons vers les 9 haut-parleurs.

Figure 4. Interface de contrôle de la synthèse sonore

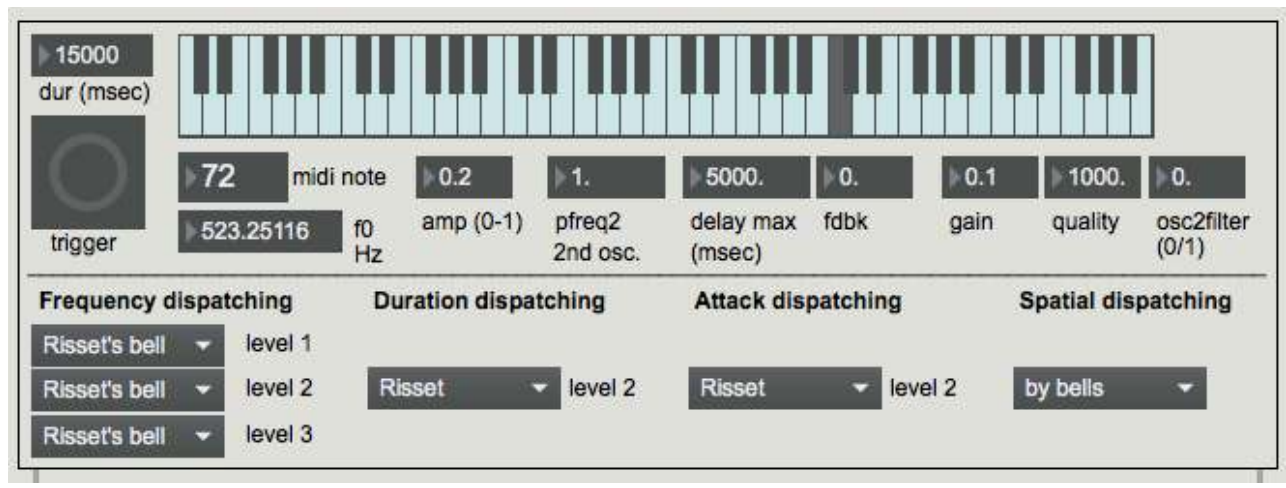

Source : auteur

Les questions posées de relations entre contenu sonore complexe et diffusion électroacoustique s'avèrent d'ailleurs très intéressantes du point de vue acoustique et musical, et donneront lieu à des recherches ultérieures associant synthèse et spatialisation du son au CICM (Centre de recherches en Informatique et Création Musicale, Laboratoire Musidanse) à l'Université Paris 8.

\subsection{Vers une « mise en résonance » de l'Hôtel-Dieu}

$17 \mathrm{Au}$ vu de la complexité des phénomènes mis en jeu - depuis la synthèse jusqu'à la diffusion dans un lieu singulier en termes de réverbération, des expérimentations empiriques ont été nécessaires, dans un premier temps, dans le studio ambisonique à 16 haut-parleurs du CICM à la Maison des Sciences de l'Homme Paris Nord : il s'agissait, dans un espace réduit qui n'a rien à voir en termes de taille et de rendu acoustique avec l'Hôtel-Dieu de Tonnerre, de simuler le jeu de la cloche de cloche et d'en équilibrer les composantes. Un autre objectif était de concevoir les paramètres de contrôle qui nous 
seraient utiles en situation, donc de définir ce qui serait fixé a priori et ce qui serait modulable sur place pendant les 3 jours de montage à Tonnerre fin juin 2018. La difficulté est évidemment de trouver un équilibre entre d'une part une installation préformatée et donc difficilement adaptable et de l'autre un trop grand nombre de paramètres de réglage, de ce fait peu opérants.

Une fois sur place à l'Hôtel-Dieu, comme pour un orgue, nous avons équilibré les composantes des sons pour chacune des 11 séquences en cherchant à chaque fois un compromis entre la diffusion analytique des 121 sons et la cohérence du rendu dans l'espace. Cet équilibrage n'est pas aisé, car il doit être pensé pour un auditeur "itinérant », marchant dans le lieu pour en prendre connaissance, et découvrant des composantes variées du son à l'approche de chaque haut-parleur.

En parcourant l'installation, l'écoute sera donc décomposée. Toutefois, la longue réverbération particulière $\mathrm{du}$ lieu assure le liant des textures de cette cloche de cloches. L'ensemble des 121 sons prend également en compte cette permanence des phénomènes et s'installe dans la lenteur qu'appelle la nef par ses dimensions.

\section{Perception itinérante du son et découverte de l'Hôtel-Dieu}

20 Ici se noue un autre aspect important de la « prise de site » sonore : elle est pour bonne partie «opérée » par les visiteurs. Ce sont eux qui découvriront des relations globales ou locales liant tel ou tel son à telle propriété de l'architecture du lieu. Comme le contrôle de l'installation se fait de manière statistique par des valeurs macroscopiques, certaines des relations sont imposées au niveau de la composition, alors que d'autres émergent pour le visiteur lors de son parcours dans la nef. Pour ce dernier, il s'agit de comprendre l'espace en l'écoutant, de l'arpenter pour l'entendre et le découvrir. Le dialogue de la musique et de l'écoute qui se noue habituellement au concert trouve dans l'architecture de la nef un nouveau support, un miroir sonore tendu à chacun, un appel à l'écoute, à la réflexion, au retour sur soi tout en se projetant dans cet espace.

21 Un autre élément de découverte de l'Hôtel-Dieu par l'installation, à la frontière du sonore et du visuel, est la mise en œuvre d'une perspective inversée de haut-parleurs. Comme le montre la figure 3, les sources sonores sont placées sur deux droites convergeant vers le haut-parleur 9. Le visiteur qui arrive dans l'Hôtel-Dieu entre nécessairement du côté tribune et découvre une perspective sonore et visuelle de hautparleurs dont le point de fuite, le haut-parleur 9 , est placé derrière lui. Alors que son regard converge vers le chœur et la statue de Marguerite de Bourgogne situés quelque 55 mètres face à lui, l'installation Les Songes de la nef lui propose de découvrir une perspective sonore et visuelle inversée de l'Hôtel-Dieu.

Envisagée de cette manière, la prise de site n'est pas l'occupation ou le remplissage du lieu, mais bien la création d'un réseau de relations, statiques ou dynamiques, entre l'installation Les Songes de la nef et l'Hôtel-Dieu, par la construction d'un instrument dont le parcours sensible permet de découvrir ces interactions. 


\section{BIBLIOGRAPHIE}

Déotte Jean-Louis, " Prise de site » ", Appareil [En ligne], Galerie, mis en ligne le 20 juillet 2018, consulté le 25 septembre 2018. URL : http://journals.openedition.org/appareil/2361; DOI :

10.4000/appareil.2361

- « Le Grand Voile de Caroline Coppey, œuvre in situ », Appareil [En ligne], Galerie, mis en ligne le 06 septembre 2018, consulté le 26 septembre 2018. URL : http://journals.openedition.org/ appareil/2179 ; DOI : 10.4000/appareil.2179

- «Éditorial », Appareil [En ligne], 15 | 2015, mis en ligne le 16 juillet 2015, consulté le 26 septembre 2018. URL : http://journals.openedition.org/appareil/2181

- Le Musée, l'origine de l'esthétique, Paris : L'Harmattan, 1993.

Lachenmann Helmut, Écrits et entretiens, nouvelle édition, textes choisis et préfacés par Martin Kaltenecker, traduits par Nicolas Donin et al., Genève, Éditions Contrechamps, 2009, 286 pages.

Risset Jean-Claude, An Introductory Catalogue of Computer Synthesized Sounds, New Jersey, Murray Hill, 1969.

\section{Références sonores}

Risset Jean-Claude, Songes, 1979, Neuilly-sur-Seine, auditorium de la SACEM.

\section{NOTES}

1. L'installation a été présentée à l'Hôtel-Dieu de Tonnerre en Bourgogne du $1^{\mathrm{er}}$ juillet au 2 septembre 2018. Une présentation de ses principes et des extraits sonores sont proposés à l'adresse : http://www.alainbonardi.net/songes.html [lien vérifié le 5 août 2018].

2. À l'initiative de la commande de cette installation par l'association Art et Patrimoine Hospitalier en Tonnerrois (APHT).

3. Déotte Jean-Louis, « Prise de site » », Appareil [En ligne], Galerie, p. 1, mis en ligne le 20 juillet 2018, consulté le 25 septembre 2018. URL : http://journals.openedition.org/appareil/2361 ; DOI : 10.4000/appareil.2361

4. Ibid., p. 1.

5. Déotte Jean-Louis, « Le musée, un appareil universel », Appareil [En ligne], Articles, p. 1, mis en ligne le 17 mars 2008, consulté le 25 septembre 2018. URL: http://journals.openedition.org/ appareil/302; DOI : 10.4000/appareil.302

6. Le mot « critique » n'a pas ici de sens négatif.

7. Berio Luciano, Entretiens avec Rossana Dalmonte, Genève, Éditions Contrechamps, 2010, 192 pages.

8. Lachenmann Helmut, «De la composition» (1986), in Écrits et entretiens, textes choisis et préfacés par Martin Kaltenecker, traduits par Nicolas Donin et al., Genève, Éditions Contrechamps, 2009, p. 134.

9. Alors qu'il n'a jamais composé pour cet instrument.

10. Lachenmann Helmut, « De la composition » (1986), in Écrits et entretiens, op. cit., p. 136.

11. Le nom de l'installation, Les Songes de la nef, renvoie à l'œuvre pour bande magnétique 4 pistes de Jean-Claude Risset intitulée Songes (1979). Cette pièce associe des sons enregistrés d'instruments à des sons de synthèse par ordinateur, notamment des sons de cloches, utilisés 
comme liaison entre les deux mondes, de l'instrumental à l'électronique, et au-delà de la réalité au rêve.

12. Risset Jean-Claude, An Introductory Catalogue of Computer Synthesized Sounds, New Jersey, Murray Hill, 1969.

13. Notons que la fréquence $\mathrm{f}_{0}$ de base du calcul, ici $440 \mathrm{~Hz}$, n'est pas directement entendue dans le son complexe. On entend en revanche deux partiels à $2 \mathrm{xf}_{0}$ et $3 \mathrm{x} \mathrm{f}_{0}$.

14. Ce logiciel est en fait un patch construit avec le logiciel de transformation du son en temps réel Max. Ce dernier est fondé sur un langage de programmation graphique permettant de construire des traitements du son à base de représentations d'objets et de connexions entre eux.

\section{RÉSUMÉS}

Dans cet article, nous développons le concept de " prise de site " au niveau musical en dialogue avec Jean-Louis Déotte et ses écrits, dans le cadre de notre installation sonore Les Songes de la nef (2018) pour l'Hôtel-Dieu de Tonnerre, double hommage au philosophe et au compositeur JeanClaude Risset. À l'opposé de l'accrochage dans un musée, la " prise de site " permet de penser l'accueil d'œuvres contemporaines dans des lieux patrimoniaux comme relation dynamique entre les deux, comme une "critique » au sens du commentaire à distance entre musiques que propose le compositeur Luciano Berio. Nous avons pensé l'installation sonore Les Songes de la nef avant tout comme un instrument à construire, nous référant au compositeur Helmut Lachenmann. Nous montrons que l'installation doit être considérée comme un cadre posant un certain nombre de relations, d'abord acoustiques avec le lieu l'accueillant ; mais aussi permettant la découverte par le visiteur itinérant de relations entre le contenu sonore et l'Hôtel-Dieu, et donc une nouvelle appréhension de cet édifice patrimonial.

In this article, we develop the concept of "site taking" on a musical perspective, discussing with Jean-Louis Déotte and his writings, in the framework of our sound installation Les Songes de la nef (2018) for the the Hôtel-Dieu in Tonnerre, double tribute to the philosopher and to composer Jean-Claude Risset. Opposite to the hanging up, the "site taking" enables to think of the reception of contemporary works in heritage monuments as a dynamical relationship between both, as a "criticism" in the meaning of a remote comment between musics proposed by composer Luciano Berio. We have designed the sound installation Les Songes de la nef first of all as an instrument to build, referring to composer Helmut Lachenmann. We show that the installation is to be considered as a framework setting a certain number of relationships with the place, acoustic ones first of all. It also enables wandering visitors to discover relationships between the sound content and the Hôtel-Dieu, and therefore get a new understanding of this monument.

\section{INDEX}

Mots-clés : prise de site, installation sonore, instrument, acoustique, synthèse, modèle instrumental 


\section{AUTEUR}

\section{ALAIN BONARDI}

Maître de Conférences HDR en Informatique et Création Musicale au Département Musique de l'Université Paris 8 ; Centre de Recherche en Informatique et Création Musicale (CICM ;

Laboratoire Musidanse, EA 1572 ; alain.bonardi@univ-paris8.fr 